\title{
More errors in errors
}

SIR - The Commentary by Menger and Haim (Nature 359, 666; 1992) calls for my response as editor of the Journal of the American Chemical Society (JACS). Menger and Haim complain about the peer-review process and the "considerable anguish" they suffered during consideration of their manuscripts by JACS. I am sure many authors could write more interesting and valid discussions about the problems they have had getting their work published. Most scientists, however, are sufficiently mature and amicable that they do not feel it necessary to document for all to see their tribulations in their interactions with their peers and editors.

Since the Commentary dealt with correction of errors, it is appropriate to point out some of Menger and Haim's own errors. For example, they state that "events began with the publication of two Articles", while in fact the main paper in question was a "Communication". This is more than a technical point. JACS makes a clear distinction between Articles and Communications. In our Notice to Authors (J. Am. Chem. Soc. 113, 10A; 1991), Communications are defined as reports (usually preliminary) that are limited in length to about 1,000 words. This Notice to Authors, incidentally, also states that "Notes" and "Comments" are not published in JACS. Thus, Menger's and Haim's papers submitted to JACS were largely commenting, without the presentation of any new results, ideas or concepts, on a brief communication limited by its size to the amount of experimental detail or discussion that it could contain.

It is JACS policy that we do not publish minor corrections as separate publications that do not contain new results. (Authors can, however, publish corrections in a separate Additions and Corrections section.) JACS has a fixed page budget which is heavily oversubscribed. If a three-page correction is published, then two communications containing original new ideas will not be. Corrections should be published if the point made is important. However, in the view of the reviewers and the editor, Menger's and Haim's work fell in the category of minor corrections and did not seem to affect the basic ideas of the Breslow et al. work. I ultimately decided to publish Haim's manuscript, after considerable revision based on valuable comments by reviewers and the editor, so it is difficult to see why he is complaining.

No pressure was brought to bear on the JACS editors not to publish the Menger and Haim manuscripts. Indeed, the only attempt to influence the edito- rial process outside the usual channels was made by Menger, when he contacted the ACS publications committee and the ACS president. The ACS rightly maintains a hands-off approach in the management of its journals; so these attempts had no effect.

Finally, one can question the propriety of publishing (sometimes out of context) reviewer's comments and the contents of private correspondence. Overall, I am afraid the Commentary says more about its authors and the journal that agreed to publish it than it does about the peerreview process.

Allen J. Bard

(Editor, Journal of the

American Chemical Society)

Department of Chemistry,

University of Texas,

Austin, Texas 78712, USA

\section{Freedom to explore}

SIR - I agree with the author of the recent leading article "High time for the circuses to stop", who points out that we will continue to be enriched by increasing our knowledge of the Solar System (Nature 358, 609; 1992). However, I disagree with his assessment of the tools needed in this endeavour. Space Station Freedom will be a pivotal tool - one in a progression of tools dedicated towards this goal.

A common complaint among the uninformed it that Space Station Freedom won't do science. I ask the readers of Nature to look at the building where their laboratories are located. Does the building itself do science? No - it is how the building is fitted with equipment and how that equipment exchanges data with other researchers that makes it possible for research to be performed. Space Station Freedom is designed to be a research facility in space capable of being reconfigured over its 30-year lifetime to meet an ever-changing mix of basic and applied research.

By visiting space we are exploring a new frontier. The first explorers of terrestrial continents brought back not only gold and commercial materials, but many new plant and animal specimens. As exploration progressed, the capabilities of exploration vessels advanced. The overtly exploratory travels of Marco Polo, Charles Darwin, and Lewis and Clark, and the considerable scientific data produced as the direct result of their expeditions, show what can happen when curious people are sent to examine the frontier.

A gravitational field of $1 \mathrm{G}$ is the single environmental factor to have remained unchanged throughout life's tenure on this planet. To perform long-term research in the absence of gravity, one must go into space with a permanent laboratory. Chronic removal of that evolutionary constant will most certainly provide new insight into the ontogeny and phylogeny of life on Earth.

The lack of gravity also has a profound effect upon many physical processes. Convection, buoyancy and thermal stratification do not occur. In addition, comparatively weak physical interactions normally masked by stronger phenomena often prevail in microgravity. We have only begun to understand the effects of this novel environment on metallurgical, chemical and combustion processes. Some of the more promising results are in organic and inorganic crystallography. It is impossible to predict what new technologies and new insights will come from space research. But as new knowledge is gathered, they will surely come.

Finally, Nature's leading article suggests that Space Station Freedom might become a circus. To this challenge I respond "long live the circus". A circus, by dictionary definition, is a "pageant featuring acts of skill and daring". Such is the exploration of space.

\section{Robert W. Phillips}

Chief Scientist,

Space Station Freedom,

National Aeronautics

and Space Administration,

Washington, DC 20546, USA

\section{Photofat}

SIR - We feel it necessary to comment on Daedalus's column (Nature 359, 20; 1992) concerning breast enlargement for women. Does Daedalus believe that such new and improved women will have anything to do with average men? Ha! Such women will require new and improved men as well. Unfortunately, Photofat will not work, as the parts of men's bodies in need of enlargement contain few fat cells. We're sure Daedalus will agree with the necessity of a comparable enlargement scheme for men, considering the puniness of the average man's muscles and other parts. We assume he will not object to the frivolous alteration of a healthy, normal male body, since he has no trouble imagining this for women.

Susan M. Purcell

Virginetta S. Cannon

Carmen R. Domingo

S. B. Minsuk

Department of Molecular and Cell Biology,

315 Life Sciences Addition,

University of California,

Berkeley, California 94720, USA

NATURE · VOL 360 • 3 DECEMBER 1992 